\title{
Entry Screening at Airport as a COVID-19 Surveillance Tool: Evaluation of Thiruvananthapuram International Airport in Kerala, India
}

\author{
Chintha Sujatha ${ }^{1 *(\oplus,}$, Sreejith Lalitha Krishnankutty ${ }^{2}$, Khalid Khader $^{3}{ }^{\circledR}$, Anju K. Kanmani ${ }^{4}$, Arya Rahul ${ }^{5}$, \\ Meenu M. Suresh ${ }^{5}{ }^{\circledR}$, Reshma Rajan Sudha ${ }^{5}$, Pillaveettil Sathyadas Indu ${ }^{5}$ \\ ${ }^{1}$ Department of Community Medicine, Government Medical College, Thiruvananthapuram, Kerala, India \\ ${ }^{2}$ Airport Health Screening and Assistant Surgeon, District Medical Office, Thiruvananthapuram, Kerala, India \\ ${ }^{3}$ Physician Quality Improvements Lead, Modern Hospitals, Kodungallur, Kerala, India \\ ${ }^{4}$ Airport Health Screening, District Medical Office, Thiruvananthapuram and Junior consultant in Paediatrics, Taluk \\ Hospital, Thiruvananthapuram, Kerala, India \\ ${ }^{5}$ Department of Community Medicine, Government Medical College, Thiruvananthapuram, Kerala, India
}

Corresponding Author: Chintha Sujatha, MD, Associate Professor, Department of Community Medicine, Government Medical College, Thiruvananthapuram, Kerala, India. PIN- 695011, Tel: +91-9447375532, Email: sujathachintha@gmail.com

Received March 13, 2021; Accepted June 4, 2021; Online Published June 9, 2021

\begin{abstract}
Introduction: As part of coronavirus disease 2019 (COVID-19) control strategies, entry screening was established at International airports. An assessment of the screening system will inform decision-making for improving entry screening for infectious diseases.

Methods: Assessment of entry screening at Thiruvananthapuram international airport done during pre and post-lockdown phases. Observation, interviews, and secondary data analysis were the methods employed. The number of passengers screened, their symptom profile, the yield of screening, actions taken, staff pattern, perceptions, training, and infection control practices assessed. Chi-square test and $t$ test were used for testing significance.

Results: Out of the 46139 passengers screened pre-lockdown, $297(0.64 \%)$ had symptoms, $23(0.05 \%)$ were positive in thermal screening. Six (2\%) among them tested positive for COVID-19. Out of the 44263 passengers screened post lockdown, from May to July 2020,671 $(1.5 \%)$ were symptomatic, and $12(0.03 \%)$ were positive in thermal screening. COVID-19 was confirmed in $45(6.7 \%)$ patients identified through the screening. With the surge in cases, the proportion of passengers opting for institutional quarantine increased significantly $(P<0.001)$. None of the staff contracted the disease. Infection control practices followed by them were optimal.

Conclusion: Though the yield of thermal and symptom screening is low, entry screening is an opportunity to identify travelers at risk of COVID-19 infection. In addition, it helps in raising awareness to ensure quarantine and guides public health authorities in preventing disease spread to the community.

Keywords: Airport Entry Screening, COVID-19 Surveillance, Infection Control, Health Practices
\end{abstract}

Citation: Sujatha C, Krishnankutty SL, Khader K, Kanmani AK, Rahul A, Suresh MM, et al. Entry screening at airport as a COVID-19 surveillance tool: evaluation of thiruvananthapuram international airport in Kerala, India. Int J Travel Med Glob Health. 2021;9(2):78-83. doi:10.34172/ijtmgh.2021.13.

\section{Introduction}

The coronavirus disease 2019 (COVID-19) outbreak was declared a pandemic by the 11th of March, 2020. Airport surveillance and screening of travelers is a control strategy documented in the severe acute respiratory syndrome (SARS) epidemic in 2003 and the H1N1 outbreak in 2001. ${ }^{1}$ COVID-19 is a disease that spreads from symptomatic, pre-symptomatic, asymptomatic patients or environmental surfaces. ${ }^{2}$ Patients can be asymptomatic or present with a range of symptoms. ${ }^{3}$ Effectiveness of entry screening at airports as a control measure has its limitations like a recent exposure not progressing to symptoms ${ }^{4-6}$ symptomatic traveler without fever ${ }^{7-10}$ decreased sensitivity of non-contact infrared thermometer (NCIT) used for fever screening. ${ }^{11,12}$ Fever screening, concealing symptoms through medications ${ }^{12}$ and the non-specificity of fever as a symptom for screening. ${ }^{6,13-14}$ During the early phase of the epidemic in India, states like Kerala had to overcome many new challenges due to the novel nature of the disease, along with already known or documented challenges reported in airport screening in other epidemics. ${ }^{15,16}$

More than $80 \%$ of reported COVID-19 cases were imported in the pre-lockdown phase of the epidemic in Kerala. ${ }^{17}$ Entry

Copyright $(\subset 2021$ The Author(s). This is an open-access article distributed under the terms of the Creative Commons Attribution License (http:// creativecommons.org/licenses/by/4.0), which permits unrestricted use, distribution, and reproduction in any medium, provided the original work is properly cited. 
screening for COVID 19 was established at all international airports by the end of January 2020, when there was an inflow of passengers from the then epicenter, Wuhan. The first COVID-19 positive cases reported in Kerala belonged to this initial cohort of passengers from China. Spike of cases with the progress of pandemic globally led to strengthening and streamlining screening and surveillance at all airports in Kerala from the 1st of March. The nationwide lockdown was declared on the 24th of March, 2020. Kerala could bring down its daily reported new case count to zero by the $3 \mathrm{rd}$ of May with robust pandemic control measures. ${ }^{17}$ Flight services for expatriates restarted by the first week of May. An influx of expatriates marked the Post-lockdown phase into the state from various countries. The repatriation flights were scheduled from the 7th of May, 2020, under the 'Vande Bharat Mission' by the government of India; ${ }^{18}$ also there were commercial flights in the later period. An estimated number of more than 2.1 million non-resident Keralites live in other countries. ${ }^{19}$ Relaxing the lockdown demanded efficient strategies to prevent all possible transmissions and generation of epicenters. While there was a list of countries considered COVID-19 high risk for screening travelers in the pre-lockdown phase, every country was considered potentially high risk in the post-lockdown period in Kerala. The need for documented evidence regarding entry screening as a control strategy for COVID-19 during different phases of the epidemic and evaluating this newly established system of airport screening can inform decisions regarding future use of screening strategies in pandemic control. The objective of this study was to assess entry screening for COVID-19 at Thiruvananthapuram International airport in terms of identifying symptomatic and confirmed cases, actions taken, and infection control practices.

\section{Methods}

\section{Study Setting}

Thiruvananthapuram International Airport, located at $8.4834^{\circ} \mathrm{N}, 76.9198^{\circ} \mathrm{E}$, is one of the major airports in South India and one among the four airports in Kerala. It is located in the capital city and is the only airport in South Kerala, the region with an estimated emigrant population of $790852 .{ }^{19}$ It is the second busiest airport in Kerala, which handles more than four million passengers a year. Evaluation of entry screening system for COVID-19 at Thiruvananthapuram International Airport was done by a team of public health experts from State Prevention of Epidemics and Infectious diseases cell, Kerala, during the pre-lockdown period from March 1-24, 2020, and the post-lockdown period from the 13th of May to the 31st of July. By the latter half of July, Kerala entered the phase of community transmission of COVID-19. The 25th of July was the date on which cumulative cases due to local transmission overtook imported cases.

\section{Study Design and Sampling}

A descriptive study with a mixed approach was done. Interviews and nonparticipant observation was done for primary data collection. Secondary data analysis was done on both airport screening data and COVID-19 surveillance data. For selecting participants for interviews, simple random sampling using computer-generated random numbers was done on the list of travelers and staff. For Infection control practices, $60 \%$ was the compliance found among both staff and travelers in a pilot study. With that information sample size for interviews was calculated using the formula for

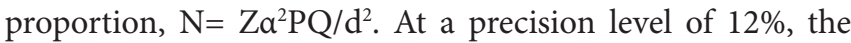
sample size needed was 66 . The sample size for secondary data analysis to obtain the yield of screening was 784 . The formula for proportion was used with an expected parameter value of 2\% (Airport surveillance data for March, lower than $6 \%-10 \%$ reported in the literature) and a precision of $1 \%$.

\section{Data Collection}

The team visited the airport and made observations using a facility and services checklist developed following guidelines issued by the Department of Health and family welfare, Kerala Government. Airport health officers, airport managers, program officers at the District Medical Officer (DMO), passengers, and personnel on duty, including doctors, nurses, health inspectors, security staff, and volunteers, were interviewed using a structured interview guide. Those persons not willing to participate in the study were excluded. Data on the number of passengers screened and their symptom profile, the yield of screening, actions taken, staff pattern, training, Personal protective equipment availability, and completeness of reporting was extracted from the self-reporting forms (SRF) and daily reports using a structured data extraction form. In addition, information regarding stakeholder perceptions, communication about infection control practices to passengers, and challenges in implementation was obtained through interviews.

\section{Statistical Analysis}

Data analysis with descriptive methodology was done using Microsoft Excel. The yield of screening and other categorical variables were expressed as proportions. Counts and mean were used for summarizing quantitative variables. For comparing the mean of passengers screened per day student $t$ test was used. Chi-square test was used for testing significance of association between quarantine locations opted and phase of the epidemic.

\section{Results}

Pre-lockdown and post-lockdown epidemiologic situations and hence the context of entry screening and methodology were different. So, the results for both phases are presented separately.

\section{Pre-lockdown Phase}

As the pandemic spread became more apparent, around 443 flights and 46139 passengers were screened at the international airport, an average number of flights and passengers per day being around 20 and 2000, respectively. The primary objective of the screening process was the isolation of suspected cases, confirming the diagnosis through laboratory investigations 
and quarantine of patients either in state-run facilities or home quarantine through appropriate screening and counseling. Out of the total 46139 passengers screened during this period, $23(0.05 \%)$ were positive in thermal screening, and a total of 297 (0.64\%) passengers had COVID-19 symptoms. Therefore, they were shifted to COVID isolation centers of Medical College or General Hospital. In March, 112 (0.24\%) passengers were moved to state-run institutional quarantine centers, as passengers returning from high-risk countries needed mandatory quarantine following the revised state guidelines. The rest of the 45842 (99.35\%) passengers were advised to remain in home quarantine for 28 days. Only 6 (2\%) among 297 symptomatic passengers tested positive for COVID-19. Details of entry screening in the pre-lockdown phase are given in Table 1. In the initial phase, asymptomatic patients under home quarantine were tested later, and the data collected in the airport facilitated contact tracing of the positives. Lockdown was declared on the 24th of March when the number of COVID-19 positive cases in Kerala was 109, of which $88(80.7 \%)$ patients were imported, and 21 (19.3\%) cases were due to local transmission.

Doctors and health staff from the state health department, in coordination with airport health officers, were effectively performing the screening activity through processes like inflight announcements and distribution of SRF, queue management, implementation of infection control practices at arrival halls, screening at the health desk with NCIT and symptom checklists, data collection using using SRF, health communications for strict quarantine, isolation of suspected cases in isolation bays and transport to COVID-19 treatment facilities through designated ambulances, and strict infection control practices at immigration and baggage. Maintaining a six-foot distance between disembarking passengers and isolation of symptomatic passengers was ensured by the cabin crew. Crowding was an issue in peak hours, especially when 2-3 flights arrived together with more than 600 passengers using the arrival hall. More than half of the passengers were non-co-operative and not maintaining the one-meter social distancing in arrival halls, a potentially high-risk area for infection spread. The use of hand sanitizers was initially variable in stations, including the SRF filling area and health desks, until strict adherence policies were established. During the initial phase, masks were worn by just $10 \%$ of the passengers. This improved to around $80 \%$ by the end of March. The Central Industrial Security Force's initial

Table 1. Entry Screening Details for COVID 19 at Thiruvananthapuram International Airport From 29-01-2020 to 24-03-2020 (Pre-lockdown)

\begin{tabular}{lcc}
\hline Activity & $\begin{array}{c}\text { Numbers From } \\
\text { 1 March }\end{array}$ & $\begin{array}{c}\text { Numbers From } \\
\text { 29 January }\end{array}$ \\
\hline Flights screened & 443 & 1032 \\
Passengers screened (SRF) & 46139 & 113413 \\
Positive in thermal screening & $23(0.5 \%)$ & 34 \\
$\begin{array}{l}\text { Referred to COVID-19 isolation } \\
\text { unit }\end{array}$ & $185(0.4 \%)$ & $201(0.17 \%)$ \\
Referred to COVID-19 care centers & $112(0.24 \%)$ & 112 \\
\hline
\end{tabular}

security and queue management were inadequate; this was later strengthened with the state police and resulted in better passenger compliance.

The arrival hall was modified to include health desks, each with two health staff, a doctor, and a nurse or junior health inspector. Screening criteria included elevated temperature, history of exposure, self-reporting, or observed signs and symptoms. Mandatory temperature screening using NCIT was augmented with a thorough history and clinical examination by the staff in the health desk as asymptomatic, pre-symptomatic, and patients on antipyretics could be identified effectively. Pamphlets were distributed to passengers with health education content and instructions regarding quarantine and prevention of the spread of COVID-19. The Ministry of Health and Family welfare guidelines mandated all international passengers to fill the SRF in the prescribed format with a duplicate copy, assisting in risk assessment, identifying suspected cases, and surveillance of positive cases. The SRF collects metadata like flight, journey details, details of cities and countries visited in the last 28 days, history of symptoms, and contact details. The original SRF form, which can be completed in three minutes, is collected at the health desk during screening and the presentation of stamped duplicate mandatory for immigration clearance. The compliance to the completion of SRF was around $60 \%$ which detailed inflight announcements and facilitation can increase. Symptomatic travelers were shifted to an isolation bay or quarantine room with an ambulance service to transport COVID hospitals and asymptomatic high-risk individuals to institutional quarantine centers. Shifting of isolated cases followed adequate infection control practices, including red channeling through a separate pathway, use of personal protective equipment (PPE) by the accompanying person with the provision of gloves, masks, and hand sanitizers.

The team set up by the Ministry of Health and Family Welfare, Government of India included four Airport Health Officers and one Health Inspector. In addition, Doctors, Nurses, Field staff, Clerical staff, Public Relation Officers, Ambulance drivers, and Data entry operators were posted by the DMO and the National Health Mission (NHM), which totaled 60 personnel for March. Police officers, volunteers, and airport staff, including cleaning staff, supported the teams, rotated in three shifts per day. No cases were reported in the airport team over this period. Reinforcement of infection control practices was done through training and communication on the importance of hand hygiene, social distancing, and PPE. All personnel used N95 masks and gloves, including the staff in immigration, customs, security counters, and cleaning. PPE was used by health staff accompanying COVID suspected passengers with proper guidelines regarding appropriate PPE for each duty station. Automatic scanners at security/ customs check minimized the risk of cross-infection using PPE mandatory for physical checking. The adherence to strict safety precautions was variable due to logistics like the burden of passengers, prolonged duty hours, and differences in self-perception of risks. Floors, surfaces, and handrails were cleaned twice daily using protective gears sealed in yellow 
bags for centralized disposal. The state government supplied PPE, masks, gloves, and sanitizers and available adequately.

\section{Post-lockdown Phase}

The post-lockdown airport surveillance activities included strengthening and streamlining all the screening activities and infection control measures practiced in the pre-lockdown period and new strategies. From the 13th of May to the 31st of July, 251 flights and 44263 passengers were screened at the international airport with an average of 3 flights and 550 passengers per day. There was a statistically significant decrease $(P<0.001)$ in average daily passengers screened during the post lockdown period, with the mean (SD) being 553.28 (88.54) when compared with the pre-lockdown period where the mean $(\mathrm{SD})$ of passengers screened per day was 2025.23 (98.76).

The screening process is aimed at isolating suspected cases and confirming the diagnosis through appropriate investigations. A walk-in thermal scanner was established instead of handheld infrared thermometers. All the international travelers had to follow the state protocol of mandatory home/institutional quarantine for 14 days, and hospital isolation was arranged for all symptomatic travelers. Out of the total 44263 passengers screened during this period, $671(1.5 \%)$ were symptomatic. Only 12 passengers were positive in thermal screening $(0.03 \%)$.

Institutional quarantine was advised if an adequate facility for room quarantine was not available at home. In the early part of post lockdown higher proportion (38.8\%) of passengers opted for home quarantine. With the rise in the number of COVID 19 cases in the state, the proportion of passengers sent to institutional quarantine increased from $59.4 \%$ in May to $81.9 \%$ by July. The proportion of hospitalized passengers as they were symptomatic also increased from $1.5 \%$ to $1.6 \%$. This difference in quarantine location opted was statistically significant $(P<0.001)$. Details of entry screening for COVID-19 at Thiruvananthapuram International Airport and comparison of quarantine location from May 13, 2020 to July 31, 2020 are provided in Table 2.

Testing became a priority to prevent the importation of cases into a population that has flattened its epidemic curve and reported zero cases in the initial days of May. Symptomatic travelers who were shifted to hospitals underwent reverse transcription polymerase chain reaction (RT-PCR) tests. COVID-19 was confirmed in 45 (6.7\%) patients identified through symptom screening. Testing for COVID-19 IgM antibody was available from the 25th of June to the 21st of July. During this period, testing was done on all asymptomatic passengers. Of the 20473 passengers tested, 961 (4.7\%) were positive for IgM antibodies. Those found positive were tested further with RT-PCR or Antigen test to detect active infection. Sixty-nine (7.2\%) patients had a positive test on confirmation. Antigen testing for COVID-19 started on the 25th of June, and it was included in Airport entry screening from the 2nd of July. All symptomatic passengers and those with antibody positivity underwent testing for COVID-19 antigen. During our study period, 826 antigen tests were done, out of which 57 (6.9\%) passengers were COVID-19 positive. None of the airport staff or staff on screening duty contracted the disease during the study period.

\section{Discussion}

Entry screening provided a low yield of both positive and symptomatic cases due to decreased number of infections, asymptomatic and pre-symptomatic presentations, and misleading disclosures. Documented evidence during previous epidemics as well as current pandemic verify the fact that entry screening contributes very little to the prevention of importation of cases as the positive predictive value of fever as a screening symptom is very low, ${ }^{4,13,20}$ thereby underlining the importance of social distancing, masking, hand hygiene and quarantines to prevent local transmission. The range of sensitivity of NCIT is between $80 \%-99 \%$, indicating a wide variation in missed cases (false negatives), and the specificity of NCITs ranges from $75 \%-99 \%$ resulting in false positives. ${ }^{4,21}$ In an entry risk assessment and management program instituted in US airports, during the 17th of January-the 13th of September, 2020, a total of 766044 travelers were screened, $298(0.04 \%)$ of whom met criteria for public health assessment; $35(0.005 \%)$ were tested for severe acute respiratory syndrome coronavirus 2 (SARS-CoV-2), and only nine $(0.001 \%)$ had a positive test result. ${ }^{22}$ During the pre-lockdown and post-lockdown phase, respectively, only $2 \%$ and $6.7 \%$ of symptomatic patients tested positive for COVID-19 in this study. Symptomatic accounted for $1.5 \%$ of total passengers. Only $0.05 \%$ of passengers were positive on thermal scanning, which can be improved with automatic thermal scanners and proper clinical assessment with a completed SRF, preferably through an app on personal devices. A screening app can provide immediate access to these details, which can be used

Table 2. Entry Screening Details for COVID 19 at Thiruvananthapuram International Airport From 13-05-2020 to 31-07-2020 (Post-lockdown) and Comparison of Quarantine Locations Across Months

\begin{tabular}{lcccccc}
\hline \multirow{2}{*}{ Month } & \multirow{2}{*}{ Total number of flights screened } & \multicolumn{4}{c}{ Quarantine for passengers } \\
\cline { 3 - 7 } & & $\begin{array}{c}\text { Home } \\
\text { No. }(\%)\end{array}$ & $\begin{array}{c}\text { Institutional } \\
\text { No. }(\%)\end{array}$ & $\begin{array}{c}\text { Hospital } \\
\text { No. }(\%)\end{array}$ & $\begin{array}{c}\text { Others } \\
\text { No. }(\%)\end{array}$ & Total \\
\hline May (13th to 31st) & 20 & $1428(38.8)$ & $2187(59.4)$ & $57(1.5)$ & $9(0.2)$ & 3681 \\
June & 113 & $3962(19.7)$ & $15799(78.7)$ & $287(1.43)$ & $24(0.1)$ & 20072 \\
July & 118 & $2870(13.9)$ & $16799(81.9)$ & $327(1.6)$ & $514(2.5)$ & 20510 \\
Total & 251 & $8260(18.7)$ & $34785(78.6)$ & $671(1.5)$ & $547(1.2)$ & 44263 \\
\hline
\end{tabular}

Chi-square $(6,44263)=1753.79, P<0.001$ 
for ethically driven public health decisions leading to better outcomes. An app can also prevent infection risk to the screening team and bottlenecks in airports by minimizing physical contact.

The use of fever as a screening symptom may not be effective due to its non-specificity and manipulation with improper history and medications. Only a third of patients were identified in Hong Kong and Singapore during the H1N1 epidemic, and the rest of the imported cases were identified by the local healthcare system. Entry screening acted as a check for traveling for the sick, thereby leading to increased public awareness. ${ }^{23}$ The success of the screening program was determined by various factors like the natural history of the pathogen, current epidemiological knowledge like incubation periods, transmission methods, ${ }^{24}$ and the spread through asymptomatic and pre-symptomatic. The use of the SRF and their contribution to preventing local spread are highly debatable. ${ }^{14,15,25}$

Further evidence supports departure screening as more effective than entry screening, ${ }^{20}$ but ignored due to logistic and political reasons. ${ }^{26,27}$ Travelers with recent exposure tend to be pre-symptomatic, resulting in a missed case in departure screening which may turn positive in arrival screening. Screening at the departure terminals is more costeffective and easier logistically during an outbreak as they need to be implemented only in affected regions. Hence the understanding that both departure and arrival screening has limitations in preventing the importation of cases as screening programs can miss a substantial portion of infected travelers. This should prompt policymakers to allocate enough logistic resources on contact tracing and containment, thereby mixing and matching screening and containment measures following the stage of the disease. ${ }^{5,6,28}$ Epidemiological factors like mean and variance in incubation period determine the effectiveness of fever screening as infections with short incubation periods result in symptoms during travel ${ }^{5,6,29}$ unlike the present pandemic, which has a long and variable incubation resulting in less yield. ${ }^{2430}$ Therefore, a completed SRF is much more

\section{Research Highlights}

\section{What Is Already Known?}

The sensitivity and specificity of entry screening measures, including thermal and symptom screening in identifying confirmed cases, were reported to be low for diseases like influenza $(\mathrm{H} 1 \mathrm{~N} 1)$ and SARS. In addition, recent studies were done in COVID 19 also reports low yield with airport entry screening.

\section{What Does This Study Add?}

The number of COVID 19 cases identified through thermal and symptom screening was low. Nevertheless, airport entry screening is an opportunity to identify travelers at risk of COVID-19 infection. In addition, it helps to raise awareness, ensure quarantine, and supports public health authorities in preventing community transmission. relevant than fever as a screening tool, especially in cases with a variable incubation period. ${ }^{24}$ The arrival screening at Sydney international airport during the $\mathrm{H} 1 \mathrm{~N} 1$ pandemic in 2009, which used a combination of risk factors and fever, had an empirical sensitivity of $7 \%$ (95\% CI: $1-18 \%)$. Also, questionnaire-based arrival screening for influenza A/H1N1 in Auckland, New Zealand, detected 4 of 69 infected individuals with a sensitivity of $6 \%$ (95\% CI: $2-14 \%) .{ }^{24}$ Despite the low percentages of confirmed cases identified through entry screening, the screening program has influenced outcomes by discouraging travel of ill persons, raising awareness, educating the traveling public, and maintaining the operation of flights from/to the affected areas. ${ }^{31}$ Entry screening measures can be an effective strategy in controlling the COVID-19 pandemic if combined with information strategies, testing, contact tracing, and quarantine measures.

\section{Conclusion}

The yield of confirmed COVID-19 cases from thermal and symptom screening was low. Entry screening acted as an opportunity to identify travelers at risk of COVID-19 infection, raise awareness, ensure quarantine and ultimately support public health authorities in preventing community transmission. In addition, the existence of airport screening may also deter some people with high-risk contacts or fever from traveling. Strategic planning and coordinated effort of multiple stakeholders have helped successfully implement airport entry screening as an essential strategy in Kerala's fight against this novel virus.

\section{Authors' Contributions}

CS and SK: Conceptualization, methodology, investigation, formal analysis, original draft preparation, review, and editing. KK: Conceptualization, original draft preparation, review, and editing. AK: Investigation, formal analysis, review. AR: Investigation, formal analysis, original draft preparation, review, and editing. MS: Investigation, formal analysis, review, and editing. RS: Methodology, review, and editing. PI: Conceptualization, methodology, supervision, review, and editing.

\section{Conflict of Interest Disclosures}

The authors declared no potential conflicts of interest for the research, authorship, or publication of this article.

\section{Ethical Approval}

This study was approved by the Institutional Ethics Committee of Government Medical College, Thiruvananthapuram, with a code of HEC.No.03/52/2020/MCT. Moreover, informed consent was obtained from the participants with strict confidentiality of information.

\section{Funding/Support}

This research did not receive any specific grant from funding agencies in the public, commercial, or not-for-profit sectors.

\section{References}

1. Wickramage K. Airport entry and exit screening during the Ebola virus disease outbreak in Sierra Leone, 2014 to 2016. Biomed Res Int. 2019;2019:3832790. doi:10.1155/2019/3832790.

2. Ferretti L, Wymant C, Kendall M, et al. Quantifying SARS- 
CoV-2 transmission suggests epidemic control with digital contact tracing. Science. 2020;368(6491). doi:10.1126/science. abb6936.

3. Guan WJ, Zhong NS. Clinical characteristics of COVID-19 in China. Reply. N Engl J Med. 2020;382(19):1861-1862. doi:10.1056/NEJMc2005203.

4. Quilty BJ, Clifford S, Flasche S, Eggo RM. Effectiveness of airport screening at detecting travellers infected with novel coronavirus (2019-nCoV). Euro Surveill. 2020;25(5):2000080. doi:10.2807/1560-7917.es.2020.25.5.2000080.

5. Pitman RJ, Cooper BS, Trotter CL, Gay NJ, Edmunds WJ. Entry screening for severe acute respiratory syndrome (SARS) or influenza: policy evaluation. BMJ. 2005;331(7527):1242-1243. doi:10.1136/bmj.38573.696100.3A .

6. Mabey D, Flasche S, Edmunds WJ. Airport screening for Ebola. BMJ. 2014;349:g6202. doi:10.1136/bmj.g6202 .

7. ssiri A, McGeer A, Perl TM, et al. Hospital outbreak of Middle East respiratory syndrome coronavirus. N Engl J Med. 2013;369(5):407-416. doi:10.1056/NEJMoa1306742.

8. Cao B, Li XW, Mao Y, et al. Clinical features of the initial cases of 2009 pandemic influenza A (H1N1) virus infection in China. N Engl J Med. 2009;361(26):2507-2517. doi:10.1056/ NEJMoa0906612.

9. Donnelly CA, Fisher MC, Fraser C, et al. Epidemiological and genetic analysis of severe acute respiratory syndrome. Lancet Infect Dis. 2004;4(11):672-683. doi:10.1016/s14733099(04)01173-9.

10. 10 Louie JK, Acosta M, Winter K, et al. Factors associated with death or hospitalization due to pandemic 2009 influenza A(H1N1) infection in California. JAMA. 2009;302(17):18961902. doi:10.1001/jama.2009.1583.

11. Hausfater P, Zhao Y, Defrenne S, Bonnet P, Riou B. Cutaneous infrared thermometry for detecting febrile patients. Emerg Infect Dis. 2008;14(8):1255-1258. doi:10.3201/eid1408.080059.

12. Nishiura H, Kamiya K. Fever screening during the influenza (H1N1-2009) pandemic at Narita International Airport, Japan. BMC Infect Dis. 2011;11:111. doi:10.1186/1471-2334-11-111.

13. Anderson RM, Fraser C, Ghani AC, et al. Epidemiology, transmission dynamics and control of SARS: the 20022003 epidemic. Philos Trans R Soc Lond B Biol Sci. 2004;359(1447):1091-1105. doi:10.1098/rstb.2004.1490 .

14. Gunaratnam PJ, Tobin S, Seale H, Marich A, McAnulty J. Airport arrivals screening during pandemic (H1N1) 2009 influenza in New South Wales, Australia. Med J Aust. 2014;200(5):290-292. doi:10.5694/mja13.10832.

15. Mabey D, Flasche S, Edmunds WJ. Airport screening for Ebola. BMJ. 2014;349:g6202. doi:10.1136/bmj.g6202.

16. Hale MJ, Hoskins RS, Baker MG. Screening for influenza A(H1N1)pdm09, Auckland International Airport, New Zealand. Emerg Infect Dis. 2012;18(5):866-868. doi:10.3201/ eid1805.111080.

17. WHO. WHO | Ebola outbreak 2014-2016. WHO. http://www. who.int/csr/disease/ebola/en/. Accessed July 5, 2020. Published
2014.

18. GoK Dashboard | Official Kerala COVID-19 Statistics. https:// dashboard.kerala.gov.in/daily.php. Accessed March 10, 2021.

19. Phase 1. https://www.mea.gov.in/phase-1.htm. Accessed March $10,2021$.

20. English-Vol-1_0.pdf. https://spb.kerala.gov.in/sites/default/ files/2021-01/English-Vol-1_0.pdf. Accessed March 10, 2021.

21. Clifford S, Pearson CAB, Klepac P, et al. Effectiveness of interventions targeting air travellers for delaying local outbreaks of SARS-CoV-2. J Travel Med. 2020;27(5). doi:10.1093/jtm/ taaa068.

22. Hausfater P, Zhao Y, Defrenne S, Bonnet P, Riou B. Cutaneous infrared thermometry for detecting febrile patients. Emerg Infect Dis. 2008;14(8):1255-1258. doi:10.3201/eid1408.080059.

23. Dollard P, Griffin I, Berro A, et al. Risk assessment and management of COVID-19 among travelers arriving at designated U.S. airports, January 17-September 13, 2020. MMWR Morb Mortal Wkly Rep. 2020;69(45):1681-1685. doi:10.15585/mmwr.mm6945a4.

24. Cowling BJ, Lau LL, Wu P, et al. Entry screening to delay local transmission of 2009 pandemic influenza A (H1N1). BMC Infect Dis. 2010;10:82. doi:10.1186/1471-2334-10-82.

25. Cho KS, Yoon J. Fever screening and detection of febrile arrivals at an international airport in Korea: association among self-reported fever, infrared thermal camera scanning, and tympanic temperature. Epidemiol Health. 2014;36:e2014004. doi:10.4178/epih/e2014004.

26. Bogoch, II, Creatore MI, Cetron MS, et al. Assessment of the potential for international dissemination of Ebola virus via commercial air travel during the 2014 west African outbreak. Lancet. 2015;385(9962):29-35. doi:10.1016/s01406736(14)61828-6.

27. Khan K, Eckhardt R, Brownstein JS, et al. Entry and exit screening of airline travellers during the A(H1N1) 2009 pandemic: a retrospective evaluation. Bull World Health Organ. 2013;91(5):368-376. doi:10.2471/blt.12.114777.

28. Bitar D, Goubar A, Desenclos JC. International travels and fever screening during epidemics: a literature review on the effectiveness and potential use of non-contact infrared thermometers. Euro Surveill. 2009;14(6):19115. doi:10.2807/ ese.14.06.19115-en.

29. Al-Tawfiq JA, Hinedi K, Ghandour J, et al. Middle East respiratory syndrome coronavirus: a case-control study of hospitalized patients. Clin Infect Dis. 2014;59(2):160-165. doi:10.1093/cid/ciu226.

30. Bedford J, Enria D, Giesecke J, et al. COVID-19: towards controlling of a pandemic. Lancet. 2020;395(10229):1015-1018. doi:10.1016/s0140-6736(20)30673-5.

31. Mouchtouri VA, Christoforidou EP, An der Heiden M, et al. Exit and entry screening practices for infectious diseases among travelers at points of entry: looking for evidence on public health impact. Int J Environ Res Public Health. 2019;16(23):4638. doi:10.3390/ijerph16234638. 